DE91 001148

\title{
Supernova Neutrinos, Giant Resonances, and Nucleosynthesis
}

\author{
Wick Haxton \\ Nuclear Theory Group, Department of Physics, FM-15 \\ University of Washington, Seattle, Washington 98195
}

Symposium in Honor of Akito Arima:

Nuclear Physics in the 1990's

Santa Fe, New Mexico, May 1990

\section{PREPARED FOR THE U.S. DEPARTMENT OF ENERGY}

This report was prepared as an account of work sponsored by the United States Government. Neither the United States nor the United States Department of Energy, nor any of their employees, nor any of their contractors, subcontractors, or their employees, makes any warranty, express or implied, or assumes any legal liability or responsibility for the product or process disclosed, or represents that its use would not infringe privately-owned rights.

By acceptance of this article, the publisher and/or recipient acknowledges the U.S. Government's right to retain a nonexclusive, royalty-free license in and to any copyright covering this paper.

\section{DISCLAIMER}

This report was prepared as an account of work sponsored by an agency of the United States Government. Neither the United States Government nor any agency thereof, nor any of their Goves any warranty, express or implied, or assumes eny legal liability or responsiemployees, makes any warranty, express or implied, or any information, apparatus, product, or bility for the accuracy, completeness, or usefulness of any ingrivately owned rights. Refer. process disclosed, or represents that its use would not infren service by trade name, trademark, ence herein to any specific commercial product, process, or service by trade name, tradorste manufacturer, or otherwise does not necessarily conster or any agency thereof. The views mendation, or favoring by the United States Government or any agency reflect those of the and opinions of authors expressed herein do 


\title{
SUPERNOVA NEUTRINOS, GIANT RLSONANCES, AND NUCLEOSYNTHESIS
}

\author{
Wick Haxton \\ Nuclear Theory Group, Department of Physics, FM-15 \\ University of Washington, Seattle, Washington 98195
}

\begin{abstract}
Almost all of the $3 \cdot 10^{53}$ ergs liberated in a core collapse supernova is radiated as neutrinos by the cooling neutron star. These neutrinos can excite nuclei in the mantle of the star by their neutral and charged current reactions. I argue that the resulting spallation reactions are an important nucleosynthesis mechanism that may be responsible for the galactic abundances of ${ }^{7} \mathrm{Li},{ }^{11} \mathrm{~B},{ }^{19} \mathrm{~F},{ }^{138} \mathrm{La},{ }^{180} \mathrm{Ta}$, and approximately a dozen other light nuclei.
\end{abstract}

\section{INTRODUCTION}

In this talk I will describe work performed with Stan Woosley, Dieter Hartmann, and Rob Hoffman on the neutrino process; a mechanism we suggest is responsible for important nuclesynthesis during a core-collapse supernova. ${ }^{1,2}$ During a Type II supernova approximately $3 \cdot 10^{53} \mathrm{ergs}$ is released by the cooling neutron star in the form of neutrinos of all flavors. ${ }^{3,4}$ These neutrinos interact with the nuclei in the various ejected shells of the supernova. In particular, inelastic neutral and charged current neutrino scattering off nuclei in or near their ground states can lead to excitation of particle unbound states that decay by neutron, proton, or $\alpha$-emission, e.g.

$$
(Z, A)+\nu \rightarrow(Z, A)^{*}+\nu^{\prime} \rightarrow\left\{\begin{array}{ccc}
(Z, A-1) & +n \\
(Z-1, A-1) & + & p \\
(Z-2, A-4)+ & +\alpha
\end{array}\right\}+\nu^{\prime}
$$

The higher temperature muon and tauon neutrinos, $\mathrm{T} \sim 8 \mathrm{MeV}$, are of particular importance because these neutrinos are capable of exciting both allowed and first-forbidden giant resonance states. ${ }^{5}$ [The average neutrino energy is approximately $3.15 \mathrm{~T}$. Neutrinos from the high-energy tails of the neutrino spectrum are particularly effective in exciting nuclei.] I will describe some of the neutrino physics of a supernova, and the nuclear physics and astrophysics that suggest the neutrino process is an important new mode of nucleosynthesis.

\section{THE SUPERNOVA EVENT}

A massive $\operatorname{star}\left(\sim 20 \mathrm{M}_{\odot}\right)$ is thought to proceed through its hydrostatic burning lifetime in about 10 milion years, from initial hydrogen burning to the final rapid phase of silicon burning in the high-temperature core of the star. The resulting "onion-skin" composition o: the presupernova progenitor, with successive shells of $\mathrm{H}, \mathrm{He}, \mathrm{C}, \mathrm{O}, \mathrm{Ne}, \mathrm{Si}$, and Fe, is shnwn in Fig. 1. The hydrostatic burning phase ends with the formation of an inert $\mathrm{Fe}$ core, leaving the star with no mechanism for maintaining the electron gas pressure necessary to support itself against gravity. 
The heating accompanying collapse leads to rapid photodisintegration of iron, which takes energy from the electron sea, and electron capture on free protons. Both processes accelerate the loss of gas pressure. Thus the collapse is quite rapid, about 0.6 the free fall velocity. The first interesting density regime is reached at. $\rho \sim 10^{12} \mathrm{~g} / \mathrm{cm}^{3}$, where the coherent scattering of neutrinos leads to trapping: that is, the time required for the neutrinos to diffuse out of the core is longer than the collapse timescale. After this point the energy liberated by mass falling deeper into the gravitational potential remains within the star during collapse.

The velocity of sound in nuclear matter increases with density. The inner 0.6-0.9 $\mathrm{M}_{\odot}$ of the iron core comprises the homologous core, that region where $V_{\text {sound }} \gtrsim V_{\text {infall, }}$, so that density perturbations can smooth out on the collapse time scale. Thus the homologous core maintains its density profile during collapse. At about three times nuclear density, $\sim 10^{15} \mathrm{~g} / \mathrm{cm}^{3}$, the center of the homologous core suddenly comes to rest as the nuclear equation of state fights further compression. The rebound produces a pressure wave that moves outward toward the edge of the homologous core. Successive shells repeat this process, so that a series of such waves coilect at the sonic point, the edge of the homologous core. Finally, as the edge of the homologous core reaches nuclear density, the released shock wave begins its journey through the remainder of the iron core.

While the shock wave initially may carry an order of magnitude more energy than is required to blow off the mantle of the star, it rapidly weaken as it "boils" Fe nuclei falling through the shock front, at a cost of about $8 \mathrm{MeV} /$ nucleon. Furthermore, as the shock wave approaches the trapping radius ("neutrinosphere") at about $10^{12} \mathrm{~g} / \mathrm{cm}^{3}$, neutrino losses rise rapidly: the electron capture rate in the hot nucleonic sea left in the wake of the shock wave is very rapid, and these $\nu_{e}$ 's can now more readily escape because the destruction of the Fe has greatly reduced the opacity. The penetration of the neutrinosphere by the shock wave is responsible for the "deleptonization burst" of neutrinos seen in Fig. 2.

The net result is a severe weakening of the shock wave as it proceeds through the iron core. In the "prompt explosion" model it is hoped that the shock wave can survive these losses and deliver enough energy to the mantle of the star to eject these layers. Hydrodynamic calculations even with favorable assumptions (a small 10-12 $\mathrm{M}_{\odot}$ progenitor with a soft nuclear equation of state), however, often fails to generate a successful explosion. In the "delayed explosion" model the shock wave stalls about $300 \mathrm{~km}$ from the center of the star, but is regenerated about one second later by neutrino reheating of nucleons left in the wake of the shock. While this model has encountered many difficulties over the years, there is a recent claim that inclusion of neutrino heating by $\nu+\bar{\nu} \rightarrow e^{+} e^{-}$can lead to a successful explosion. Two other groups are working to verify this prediction, but so far have not succeeded in producing an explosion.

Regardless of the explosion mechanism, theorists agree that the total number of neu* trinos and antineutrinos produced by the core collapse and subsequent cooling of a massive $\left(\sim 20 \mathrm{M}_{i}\right)$ star is about $10^{58}$. The vast majority of these neutrinos are emitted as the 
neutron star cools: the neutrino luminosity after shock breakout at the neutrinosphere is well approximated by an exponential decay curve with a time constant $\tau_{\nu} \sim 3 \mathrm{~s}$. The mean energy of the muon and tauon neutrinos is about 25 to $30 \mathrm{MeV}(\mathrm{T} \sim 8-10 \mathrm{MeV})$. The electron neutrino distributions are cooler $\left(\mathrm{T} \sim 4 \mathrm{MeV},<E_{\nu}>\sim 12 \mathrm{MeV}\right)$ because of their charged current interactions. The neutrinosphere, or approximate trapping radius, for electron neutrinos lies farther from the center of the star, where the temperature is lower. ${ }^{3,4}$

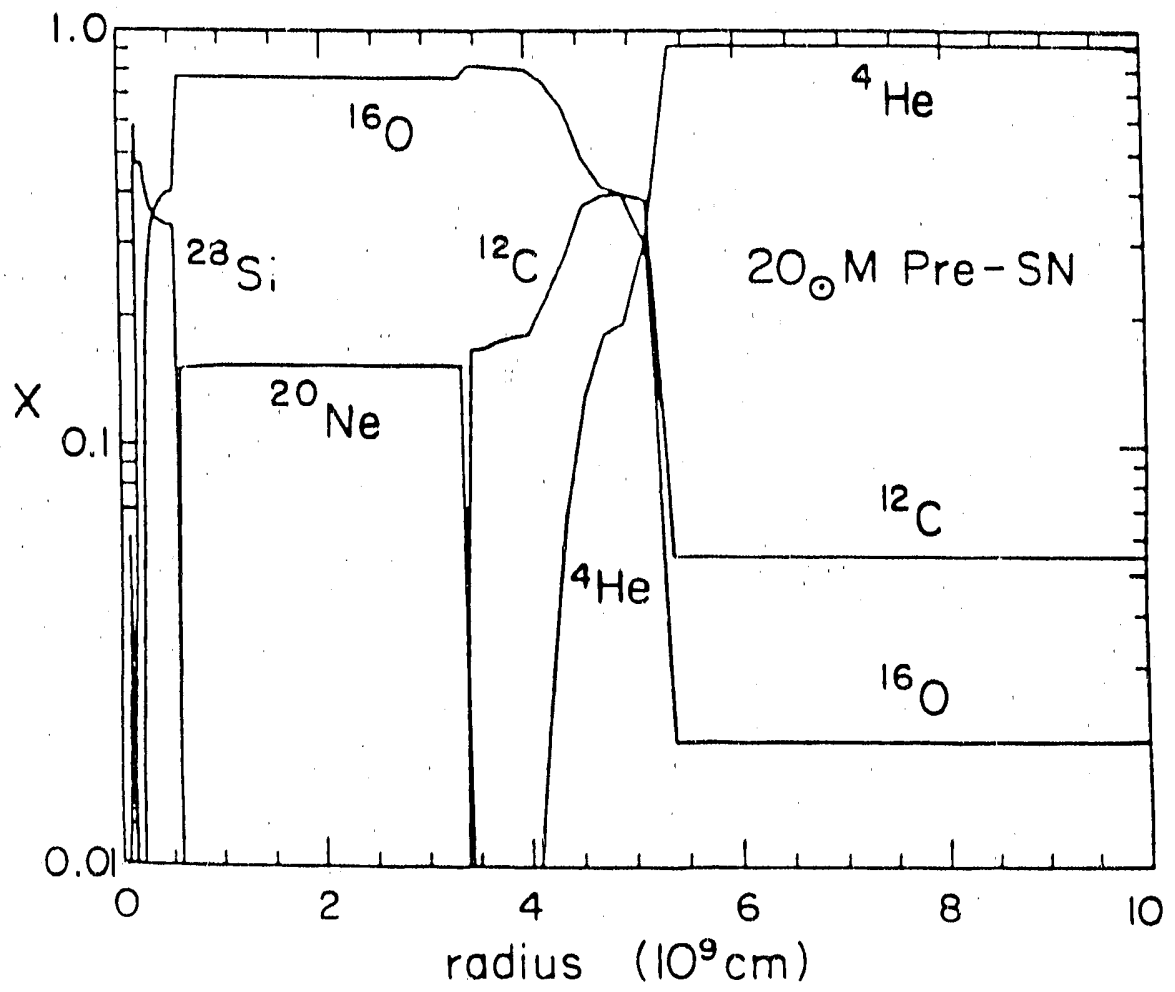

FIGURE 1

Mass fractions as a function of radius for the pre-supernova $20 \mathrm{M}$. star.

Our treatment of the neutrino process requires us to describe the radius, temperature, and density history of the various irradiated zones within the supernova. Two phases of the explosion are of interest. Prior to the arrival of the shock wave (generated by the core bounce) a stellar zone at some fixed radius experiences an exponentially declining neutrino flux at approximately constant temperature and density. This is the pre-processing phase. As the shock wave passes through this zone the temperature rises to some peak value, and the necessary overpressure is generated to drive the zone outward in a rapid expansion. In the subsequent dynamic post-processing phase the temperature and density drop rapidly as a result of the adiabatic expansion of the zone, and the radial expansion leads to an additional reduction in the effective neutrino flux. We will see that important neutrino-induced nucleosynthesis occurs in both of these phases. The initial conditions encountered in the preprocessing phase are the end result of the star's evolution through hydrostatic burning. Our explorations were carried out for a "typical" Type Il supernova progenitor of $20 \mathrm{M},{ }^{6,7}$ The specific model was that of Woosley and Weaver ${ }^{8}$, a Population 
1 presupernova star that was evolved without mass loss, including semiconvection, and using the Caughlan et al. (1985) reaction rate for ${ }^{12} \mathrm{C}(\alpha, \gamma){ }^{16} \mathrm{O} .{ }^{9}$ The star begins its lifetime burning hydrogen, with an initial composition taken from Cameron except for an increase in helium mass fraction to 0.25 (and a corresponding decrease in the hydrogen mass fraction). After hydrogen burning has finished, the resulting ashes determine the initial composition at the onset of helium burning. This process continues, with the ashes of helium burning providing the starting composition for the carbon burning phase; etc. The result, just prior to core collapse, is a star whose zones resemble an onion: an iron core surrounded by layers of silicon, oxygen, neon, carbon, helium, and hydrogen (Fig. 1).

\section{NEUTRINO-NUCLEUS INTERACTIONS}

Two years ago I pointed out that supernova modele's had omitted an important neutrino elasticity, neutral current scattering off a nucleus. ${ }^{5}$ Although all neutrino flavors participate in this process, the higher temperature muon and tauon neutrinos are more important: the reactions of energetic neutrinos are favored by phase space, particularly in cases where nuclear thresholds are high. In addition, these neutrinos can strongly excite first-forbidden transitions to giant resonances which, as discussed below, dominate cross sections at intermediate energies. ${ }^{5}$

This process, and its charged current analog, has a number of important consequences:

- During the infall stage, these semileptonic interactions and $\left(\nu_{e}, e\right)$ elastic scattering become effective "downsca tering" mechanisms by which neutrinos produced superthermally in $\beta$-decay reaction, can loose their energy. The dominating trapping mechanism, coherent scattering off nuclei, is governed by a cross section that varies as $E_{\nu}^{2}$. Thus lower energy $\nu_{e}$ 's escape the star more easily. This early radiation of lepton number leads to a small core, and thus a weaker shock. Furthermore the shock wave then has more overlying iron to penetrate. Thus this effect is very troublesome for models hoping to produce an explosion.

These qualitative arguments have been verified in the hydrodynamic calculations of Steve Bruenn.

I think there is still some hope that this early lepton number leakage may be stopped. Several reactions can be listed that have nonvanishing cross sections in the limit of zero neutrino energy: "upscattering" of a neutrino off a hot nucleus, the resonant process $\nu+$ $\bar{\nu}+A \rightarrow A^{*}$, and the analogous charged current reaction $\nu_{e}+e^{+}+A \rightarrow A^{*}$. (We assume the $\bar{\nu}$ and $e^{+}$provide energy.) Clearly, then, there is some threshold energy below which standard supernova models predict too much transparency. Whether this threshold energy' is sufficiently high to have any significant effect on lepton number loss is not yet known.

- One might expect that the inelastic scattering might help the delay explosion model by reheating undissociated nuclei (mostly ${ }^{4} \mathrm{He}$ ) lying in the shock wave's wake. However, it is easy to show that the additional effects are not large. 
- An interesting possibility is that the "deleptonization" neutrinos could "preheat" iron falling into the shock front, thus lessening the shock wave energy losses. This prediction ${ }^{5}$ was later supported by Ray and Kar, who found significant energy deposition $\left(\sim 0.7 \cdot 10^{51}\right.$ ergs) for reasonable $\nu_{e}$ temperatures of $4 \mathrm{MeV}$. However more recent work I have done in collaboration with Bruenn obtained less sanguine results, about $1 / 3$ the value above. The primary reason appears to be that more realistic neutrino diffusion calculations predict spectra that are depleted in the high energy tail relative to thermal (e.g., Fermi-Dirac) distributions.

- The most interesting results, however, involve the effect of the neutrinos on the nuclear chemistry of the star's mantle. This is the topic of the rest of this talk.

The incorporation of neutrino physics into the standard explosive nucleosynthesis reaction network required us to estimate neutrino scattering cross sections and associated particle breakup branching ratios for approximately 300 light and medium mass nuclei. We began by studying a subset of 18 "node nuclei" that included most of the network species that accumulate appreciably: ${ }^{4} \mathrm{He},{ }^{8} \mathrm{Be},{ }^{12} \mathrm{C},{ }^{14} \mathrm{~N},{ }^{16} \mathrm{O},{ }^{20} \mathrm{Ne},{ }^{24} \mathrm{Mg},{ }^{28} \mathrm{Si},{ }^{32} \mathrm{~S},{ }^{36} \mathrm{Ar}$, ${ }^{40} \mathrm{Ca},{ }^{44} \mathrm{Ti},{ }^{48} \mathrm{Cr},{ }^{52} \mathrm{Fe},{ }^{56} \mathrm{Fe},{ }^{56} \mathrm{Ni},{ }^{60} \mathrm{Zn}$, and ${ }^{80} \mathrm{Zr}$. Detailed nuclear structure studies were then undertaken to estimate the inclusive responses of these nuclei to supernova neutrinos.

The neutrino spectra were taken to be Fermi-Dirac distributions having temperatures $\mathrm{T}$ and zero chemical potential

$$
n(E)=\frac{0.555}{T^{3}} \frac{E^{2}}{e^{E / T}+1} .
$$

Calculations were performed for neutrino energies up to $150 \mathrm{MeV}$, so that accurate spectrumaveraged cross sections could be obtained for the highest temperatures of interest ( $\mathrm{T} \sim 12$ $\mathrm{MeV}$ ).

The qualitative features of the nuclear response in this energy region are well known. ${ }^{5,10}$ The threshold response is determined by allowed Gamow-Teller (GT) transitions. (The Fermi operator $T_{3}$ is diagonal and cannot generate inelastic neutral current transitions.) The allowed response is quite weak for closed-shell suclei like ${ }^{4} \mathrm{He},{ }^{16} \mathrm{O}$, and ${ }^{40} \mathrm{Ca}$, where such transitions tend to be Pauli blocked, but strong for nuclei near the middle of the $1 \mathrm{p}$, $2 s 1 d$, and $2 p 1 f$ shells. In addition, the higher energy muon and tauon neutrinos can strongly excite first-forbidden transitions to the giant resonance region. These neutrino excitations are the direct analogs of the giant E1 resonance observed in photoexcitation, generalized to include contributions from the axial current. The dominant first-forbidden operators $q_{\mu} \vec{r}(i) \tau(i)$ and $q_{\mu}(\vec{\sigma}(i) \therefore \vec{r}(i))_{J} \tau_{3}(i)(\mathrm{J}=0,1,2)$ are dependent on the momentum transfer $q_{\mu}$, and thus are enhanced for energetic neutrinos scattering in the backward direction.

In order to respect the sum rules associated with both the GT and first.forbidden operators, one must sum over "complete" sets of transitions. For GT transitions full Ohw shell model calculations yield such a complete set. In cases where the $0 \hbar \omega$ basis becomes too large, one can build a simpler model for the ground state and then sum to a complete set of $0 \hbar \omega$ one-body excitations. 
In the adopted harmonic oscillator single particle basis, first-forbidden operators connect a $0 \hbar \omega$ ground state to $1 \hbar \omega$ excitations. In several of the light nuclei we were able to complete the shell model sum to all such excitations. The net first-forbidden strength in these model calculations is often too large: in ${ }^{12} \mathrm{C},{ }^{14} \mathrm{~N}$, and ${ }^{16} \mathrm{O}$ the calculations overestimate the Thomas-Reiche-Kuhn photoabsorption sum rule, while the experimental sum over the giant resonance region undersaturates the sum rule. ${ }^{11}$ We followed many others in scaling our transition densities to correct the discrepancy, as noted below. For heavier nuclei in the 2s1d and 2p1f shells, we abandoned the shell model in favor of the simpler Goldhaber-Teller model, which satisfies the TRK sum rule and its spin generalizations.

We retained the full nuclear current operators, as described by Walecka ${ }^{10}$ and Haxton. ${ }^{5}$ Technically our calculations are then not fully inclusive, as second-forbidden and higherorder operators can connect to states outside the model spaces. Calculations carried out in an expanded model space in one case $\left({ }^{4} \mathrm{He}\right)$ verified that these contributions are negligible.

The resulting cross sections were averaged over the neutrino spectra for a range of temperatures. The results are in accord with qualitative expectations. The closed-shell nuclei $\left({ }^{4} \mathrm{He},{ }^{16} \mathrm{O},{ }^{40} \mathrm{Ca}\right)$ have smaller cross sections, a result of the weak GT strength and high thresholds for exciting isovector states. Because they are dominated by first. forbidden contributions, these cross sections have a steep $\mathrm{T}$ dependence. Nuclei in the middle of shells have substantial GT strength and thus the largest cross sections. At low $T$ these nuclei scatter neutrinos much more efficiently. However, the cross sections rise less steeply in $\mathrm{T}$ than in the case of the closed-shell nuclei. At $\mathrm{T} \sim 10 \mathrm{MeV}$ all large differences between nuclei have been smoothed out by the growing giant resonance response. Thus, at typical muon and tauon neutrino temperatures, the cross sections/nucleon $\left(\sim 10^{-42} \mathrm{~cm}^{2}\right)$ are roughly independent of the nuclear species.

As many other nuclei appear in the reaction network, a complete set of shell model calculations is not feasible. Instead, we d l loped a physically well-motivated procedure ${ }^{2}$ for interpolating the GT and giant resonance cross sections in $\mathrm{N}$ and $\mathrm{Z}$, using the 18 node nuclei results described above.

The next phase of the nuclear structure work was the evaluation of the branching ratios for the decay of the daughter nuclei. The calculations for the node nuclei produced a detailed spectrum (in one MeV bins) of the cross section as a function of nuclear excitation energy. Branching ratios appropriate for each bin were obtained from a Hauser-Feshbach optical model calculation. Nuclear level densities were taken from a statistical formula, and the allowed decay channels were assurned to be $\mathrm{n}, \mathrm{p}, \alpha, \mathrm{d},{ }^{3} \mathrm{H},{ }^{3} \mathrm{He}$, and $\gamma$. In most cases only the $\mathrm{n}, \mathrm{p}, \mathrm{a}$, and $\gamma$ channels are important. The code assumes isotopically pure nuclear states, and we neglected the small $(\sim 1 \%)$ isoscalar component in neutrino scattering. Thus the threshold for $\alpha$ emission in an $N=Z$ nucleus is determined by the position of the first isovector state in the daughter nucleus.

Developing an interpolation scheme for the remaining nuclei from the detailed HauserFeshbach studies of the node nuclei proved to be a challenge. Important differences in 
branching ratios occur from nucleus to nucleus because of rather modest shifts in thresholds. As a result, any direct interpolation based on the calculated branching ratios for node nuclei. is unreliable. Instead, we developed a simple representation for the excitation spectrum of the node nuclei, two energies and two weights chosen to reproduce the first three moments (and the normalization) of the calculated spectrum. As these parameters vary rather gently with $N$ and $Z$, we were able to estimate the corresponding moments of neighboring nuclei by interpolation. We then performed Hauser-Feshbach calculations for all of the non-node nuclei using these simplified spectra and detailed mass tables to provide correct thresholds.

The entire program described above was repeated for the charged current reactions $\left(\nu, e^{-}\right)$and $\left(\bar{\nu}, e^{+}\right)$. Note that the Fermi operator now contributes to allowed cross secrions. Coulomb effects on the electron phase space and on the positions of giant resonances (in the Goldhaber-Teller model) were taken into account. Otherwise, the procedures followed those outlined above for neutral current scattering.

\section{NEUTRINO PROCESS NUCLEOSYNTHESIS}

Detailed results for neutrino process nucleosynthesis in each of the major supernova shells and during both the preprocessing and postprocessing phases are discussed by Woosley, Hartmann, Hoffman, and Haxton. ${ }^{2}$ I cannot discuss the full results of the network calculations here. However a number of qualitative observations can be made:

1) The primary mechanism is the neutral current excitation of nuclei by muon and tauon neutrinos, though there are exceptions where charged current contributions are significant and even dominant.

2) The net result of the neutrino process is not the instantaneous production of new isotopes, but also includes all of the subsequent nuclear reactions of the coproduced protons, neutrons, alphas, etc. In some cases the net effect of these reactions is to destroy the product of interest; in others, these reactions may themselves produce new elements.

3) The products of the neutrino process may not survive the heating (and associated nuclear reactions) accompanying shock wave passage. Thus the effect of shock-wave heating is an important component of the calculations.

4) The post-processing phase is of particular importance for interior shells that expand off the neutron star at early times. Further shock-wave heating does not ocur. The composition of the shell during the neutrino illumination depends on the thermodynamic conditions extant in the hydrodynamically expanding and exponentially cooling material.

5) There are uncertainties in our calculations resulting from neutrino-nucleus cross sections, hadronic cross sections contributing to the network processing, the presupernova composition of the star, and the spectra and numbers of emitted neutrinos. We also anticipate that some differences may arise when we extend our present work, specific to a particular presupernova progenitor ${ }^{8}$, to a wider range of possible parent stars. 
The net result of our investigations is the discovery that the galactic abundances of ${ }^{7} \mathrm{Li}$, ${ }^{11} \mathrm{~B},{ }^{18} \mathrm{~F},{ }^{198} \mathrm{La}$, and ${ }^{180} \mathrm{Ta}$ appear to be due almost entirely to the $\nu$-process. Significant amounts of ${ }^{10} \mathrm{~B},{ }^{15} \mathrm{~N},{ }^{22} \mathrm{Na},{ }^{26} \mathrm{Al},{ }^{31} \mathrm{P},{ }^{35} \mathrm{Cl},{ }^{38,40} \mathrm{~K},{ }^{51} \mathrm{~V},{ }^{45} \mathrm{Sc},{ }^{48} \mathrm{Ti},{ }^{50} \mathrm{~V},{ }^{55} \mathrm{Mr}$, ${ }^{58} \mathrm{Co}$, and ${ }^{63} \mathrm{Co}$ are also produced so that, within the uncertainties of the model, these isotopes may also owe their origin predominantly to the $\nu$-process. Below I discuss the production of ${ }^{7} \mathrm{Li},{ }^{11} \mathrm{~B}$, and ${ }^{19} \mathrm{~F}$ in more detail.

The only stable isotope of fluorine, ${ }^{10} \mathrm{~F}$, has a low solar abundance relative to its neighbor, ${ }^{20} \mathrm{Ne}$, of $1 / 3100$. Thus one can account for the entire observed abundance of ${ }^{19} \mathrm{~F}$ by producing this isotope, in the same site where ${ }^{20} \mathrm{Ne}$ is synthesized, with a relative efficiency of only $0.03 \%$.

The neutral current response for ${ }^{20} \mathrm{Ne}$ is dominated by states above the threshold for particle breakup. 'The optical model predictions for the ${ }^{20} \mathrm{Ne}\left(\nu, \nu^{\prime} n\right)^{1 \theta} \mathrm{Ne}$ and ${ }^{20} \mathrm{Ne}\left(\nu, \nu^{\prime} p\right)^{1 \theta} \mathrm{F}$ branching ratios are $30 \%$ and $66 \%$ respectively. The neon rich shell, i.e., the region where carbon has at least partially burned but neon has not, lies between $(0.7-3.4) \cdot 10^{9} \mathrm{~cm}$ in our $20 \mathrm{M}$ progenitor. For a distance of $2 \cdot 10^{\circ} \mathrm{cm}$, a total energy in $\mu$ and $\tau$ neutrinos of $3 \cdot 10^{53} \mathrm{ergs}$, and an average neutrino temperature of $10 \mathrm{MeV}$, one finds that the total (instantaneous) production of mass-19 nuclei relative to ${ }^{20} \mathrm{Ne}$ parent nuclei is $0.3 \%$, or about an order of magnitude larger than the solar ratio. However, the survival of these nuclei at this point is still in doubt: both the nuclear processing of the protons and neutrons coproduced with the mass-19 nuclei and the nuclear heating when the shock wave (the ejection mechanism) passes can destroy these fragile nuclei.

The network calculations show ${ }^{1,2}$ that almost all of the ${ }^{19} \mathrm{Ne}$ is quickly destroyed, primarily by ${ }^{19} \mathrm{Ne}(n, \alpha)$. Thus this isotope does not survive to produce ${ }^{19} \mathrm{~F}$ by $\beta$ decay. The ${ }^{18} \mathrm{~F}$ can also be destroyed, with the key reaction being ${ }^{19} F(p, \alpha)^{16} \mathrm{O}$. However this process is the dominant proton reaction only if ${ }^{19} \mathrm{~F}$ is abundant. Otherwise ${ }^{23} \mathrm{Na}(p, \alpha)^{20} \mathrm{Ne}$ absorbs the protons. As the cross section for producing ${ }^{10} \mathrm{~F}$ is sharply dependent on $T_{\nu}$, the competition between these two reactions generates an interesting mechanism that prevents the overproduction of ${ }^{19} \mathrm{~F}$.

Table 1. Cross Sections, Fluences, and Nucleosynthesis of ${ }^{19} \mathrm{~F}$.

\begin{tabular}{lccccc}
\hline$T_{\nu}(\mathrm{MeV})$ & 4 & 6 & 8 & 10 & 12 \\
\hline${ }^{20} \mathrm{Ne}\left(\nu, \nu^{\prime} n\right)^{19} \mathrm{Ne}^{a}$ & 0.093 & 0.93 & 3.6 & 8.6 & 16.1 \\
${ }^{20} \mathrm{Ne}\left(\nu, \nu^{\prime} p\right)^{18} \mathrm{~F}^{a}$ & 0.25 & 2.2 & 7.9 & 18.6 & 34.4 \\
$\phi_{\text {tot }}\left(\mathrm{cm}^{-2}\right)^{b}$ & 2.96 & 1.97 & 1.48 & 1.18 & 0.99 \\
$\left.\left.{ }^{19} \mathrm{~F} /{ }^{20} \mathrm{Ne}\right]^{19} \mathrm{~F} /{ }^{20} \mathrm{Ne}\right]_{f}$ & 0.28 & 1.2 & 2.3 & 2.2 & 2.2 \\
\hline
\end{tabular}

a Cross section in $10^{-42} \mathrm{~cm}^{2}$ (average for $\nu$ and $\bar{\nu}$ )

b Total fluence $\left(10^{38} / \mathrm{cm}^{2}\right)$ of $\mu$ and $\tau$ nentrinos and antineutrinos at $2 \times 10^{4} \mathrm{~cm}$ 
The second survival factor is the peak "explosion temperature" achieved when the shock wave passes through the ${ }^{20} \mathrm{Ne}$ shell. At a radius of $10^{9} \mathrm{~cm}$ the peak temperature is about $2.5 \cdot 10^{9} \mathrm{~K}$, while at $3 \cdot 10^{9} \mathrm{~cm}$ the value is lower, about $10^{9} \mathrm{~K}$. Fluorin is efficiently destroyed by ${ }^{19} \mathrm{~F}(\gamma, \alpha)^{15} \mathrm{~N}$ for temperatures $T_{\nu} \geq 1.7 \cdot 10^{9} \mathrm{~K}$. Thus only the ${ }^{10} \mathrm{~F}$ produced at large radii can survive the shock-wave heating.

The results of Woosley and Haxton ${ }^{1}$ for the production of ${ }^{19} \mathrm{~F}$ are summarized in Table 1. (A more careful calculation appears in Ref. 2.) The quantity $\left({ }^{1 \theta} \mathrm{F} /{ }^{20} \mathrm{Ne}\right.$ ) gives the ${ }^{19} \mathrm{~F} /{ }^{20} \mathrm{Ne}$ production ratio, normalized to the solar value, after all nuclear processing has been completed, in the absence of shock-wave heating. The quantity $\left.1{ }^{18} \mathrm{~F} /{ }^{20} \mathrm{Ne}\right]_{f}$ the final ejected abundance ratio, gives the reduced value that results from including shock-wave heating.

For ternperatures near the expected value $(\sim 8 \mathrm{MeV})$ the ejected ratio is very close to that observed in the sun. Assuming an uncertainty of a factor or two in the cosmic ${ }^{19} \mathrm{~F} /{ }^{20} \mathrm{Ne}$ abundance, we also see that temperatures much below $6 \mathrm{MeV}$ appear to be ruled out, assuming that the $\nu$-process is the dominant nucleosynthesis mechanism for this element. Thus ${ }^{18} \mathrm{~F}$ serves as a crude supernova muon/tauon neutrino thermometer. Note that the growing importance of the reaction ${ }^{19} \mathrm{~F}(p, \alpha)^{16} \mathrm{O}$ prevents the overproduction of ${ }^{19} \mathrm{~F}$ for $T_{\nu}>8 \mathrm{MeV}$. The discovery of a plausible nucleosynthesis mechanism for ${ }^{19} \mathrm{~F}$ is a pleasing result.

Another classic puzzle is presented by ${ }^{11} \mathrm{~B}$. Traditionally boron has been explained as a cosmic ray spallation product. However, ${ }^{11} \mathrm{~B}$ is underproduced relative to ${ }^{10} \mathrm{~B}$ in this process, leading to the suggestion that an (otherwise) undetected low-energy component in the cosmic ray flux could preferentially produce the heavier isotope. Similar calculations to those described above suggest that this contrived solution is unnecessary: the neutrino process production of ${ }^{11} \mathrm{~B}$ by ${ }^{12} \mathrm{C}\left(\nu, \nu^{\prime} n\right)^{11} \mathrm{C} \underset{\beta}{\longrightarrow}{ }^{11} \mathrm{~B}$ in the ${ }^{12} \mathrm{C}$ shell is very robust, fully accounting for the observed abundance of this isotope.

Perhaps the most exciting result is the production of ${ }^{7} \mathrm{Li}$, an isotope of great importance because of its role in the big bang. The big bang nucleosynthesis of this element has been generally regarded as tor weak to account for the observed abundance. We find that ${ }^{7} \mathrm{Li}$ (but very little ${ }^{6} \mathrm{Li}$ ) can be made from neutral current interactions with helium in $t$ wo distinct stellar regions. Production in the helium shell, following spallaticn, occurs through ${ }^{3} \mathrm{H}(\alpha, \gamma)^{7} \mathrm{Li}$ and ${ }^{3} \mathrm{He}(\alpha, \gamma)^{7} \mathrm{Be}$. It is also produced during the post-processing phase, due to neutrino reactions with helium produced as the high-temperature ejecta cools in nuclear statistical equilibrium. In particular, the ejecta of the silicon shell experiences an alpharich freeze-cut. The total ${ }^{7} \mathrm{Li}$ production in our $20 \mathrm{M}$. model is about 0.6 of the observed abundance, which suggests that the $\nu$-process is responsible for most of the galactic ${ }^{7} \mathrm{Li}$.

\section{REFERENCES}

1) S.E. Woosley and W.C. Haxton, Nature 334, (1988) 45.

2) S.E. Woosley, D. Hartmann, R.D. Hoffman, and W.C. Haxton, submitted to Ap. J (1988). 
3) R.W. Mayle, Ph.D. thesis, Lawrence Livermore National Laboratory reprint UCRL53713 (1985); H.A. Bethe and J.R. Wilson, Ap. J. 295 (1985) 14.

4) J. Cooperstein, H.A. Bethe, and G.E. Brown, Nucl. Phys, 429 (1984) 527; E.A. Baron, J. Cooperstein, and S. Kahana, Phys. Rev. Lett. 55, (1985) 126 and Nucl Phys. A440 (1985) 744.

5) W.C. Haxton, Phys. Rev, Lett. 60 (1988) 1999.

6) W.D. Arnett and D.N. Schramm, Ap. J, Lett. 184 (1973) 247.

7) S.E. Woosley and T.A. Weaver, Ann. Rev, Astr. Astrop. 24 (1986) 205.

8) S.E. Woosley and T.A. Weaver, Phys. Rep. 163 (1988) 79.

9) G.R. Caughlan, W.A. Fowler, M.J. Harris, and B.A. Zimmerman, Atomic and Nuclear Data Tables 32 (1985) 197.

10) J.D. Walecka, in Muon Physics, Vol, 2, ed. V.W. Hughes and C.S. Wu (New York: Academic Press, 1975) p. 113.

11) E.G. Fuller, Phys. Rep 127 (1985) 185.

12) J.P. Elliott et al., Nucl. Phys. A121 (1968) 241.

13) S. Cohen and D. Kurath, Nucl. Phys. 73 (1965) 1.

14) D.J. Millener and D. Kurath, Nucl. Phys. A255 (1975) 315.

15) T.T.S. Kuo and G.E. Brown, Nucl. Phys. A114 (1968) 241.

16) T.T.S. Kuo, private communication (1985).

17) W.C. Haxton, Phys. Rev. C37 (1988) 2660.

18) T.W. Donnelly', J. Dubach, and W.C. Haxton, Nucl. Phys. A251 (1975) 353. 

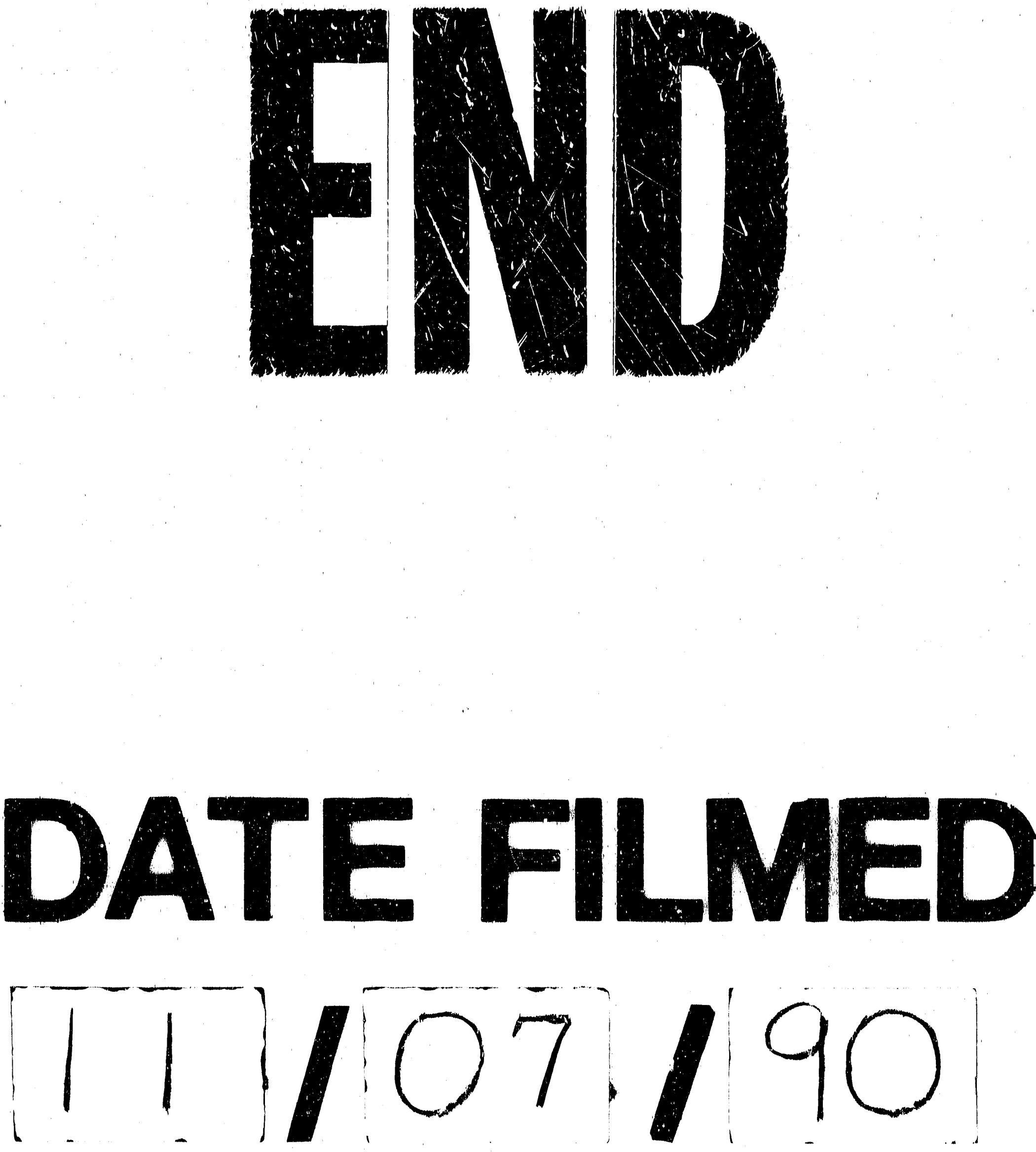